\title{
AOOT 1960
}

\section{REVUE}

\section{INTERNATIONA LE}

\author{
DE LA
}

\section{CROIX-ROUGE}

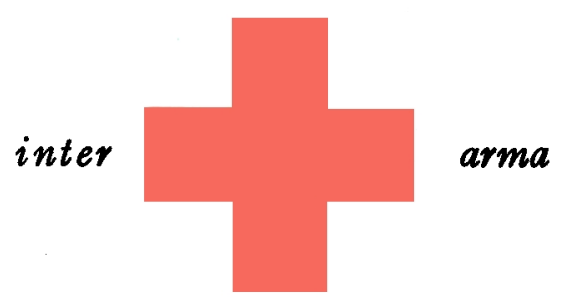

caritas

COMITÉ INTERNATIONAL DE LA CROIX-ROUGE GENEVE 


\section{COMITÉ INTERNATIONAL DE LA CROIX-ROUGE}

\section{à Genève}

MM. LEOPOLD BOISSIER, docteur en droit, professeur honoraire de l'Université de Genève, ancien secré. taire général de l'Union interparlementaire, présiden l (1946) 1

JACQUES CHENEVIERE, docteur ès lettres b.c., vice-président d'honneur (1919)

$M^{110}$ LUCIE ODIER, ex-chef du Service des infirmières-visiteuses de la Section genevoise de la Croix-Rouge suisse (1930)

MM. CARL J. BURCKHARDT, docteur en philosophie, ancien ministre de Suisse en France (1938)

MARTIN BODMER, docteur en philosophie h.c. rice-president (1940)

ERNEST GLOOR, docteur en médecine (1945)

PAUL RUEGGER, ancien ministre de Suisse en Italie et en Grande-Bretagne, membre de la Cour Permanente d'Arbitrage (1948)

RODOLFO OLGIATI, docteur en médecine h.c., ancien directeur du Don suisse (1949)

M 110 MARGUERITE VAN BERCHEM, ancien chef de service de l'Agence centrale des prisonniers de guerre (1951)

MM. FRÉDÉRIC SIORDET, avocat, conseiller du Comité international de la Croix-Rouge de 1943 a 1051, (1051)

MARCEL JUNOD, docteur en médecine, délégué du Comité international de la Croix-Rouge de 1935 à 1946, vice-président (1952)

GUILLAUME BORDIER, ingénieur dipl. E.P.F., M.B.A. Harvard, banquier (1955)

ADOLPHE FRANCESCHETTI, docteur en médecine, professeur de clinique ophtalmologique à l'Université de Genève (1958)

HANS BACHMANN, docteur en droit, secrétaire général adjoint du Comité international de la CroixRouge de 1944 à 1946 (1958)

JACQUES FREYMOND, docteur ès lettres, directeur de l'Institut universitaire de hautes études internationales, professeur à l'Université de Genève (1959)

\section{Direction :}

MM. ROGER GALLOPIN, docteur en droit, directeur excicutif

JEAN S. PICTET, docteur en droit, directeur des Affaires générales

EDOUARD de BONDELI, sous-directeur, Services financiers et administratifs

CLAUDE PILLOUD sous-directeur Service juridique

1 Les années indiquées dans les parenthèses đésignent les dates de nomination des membres du Comité international. 


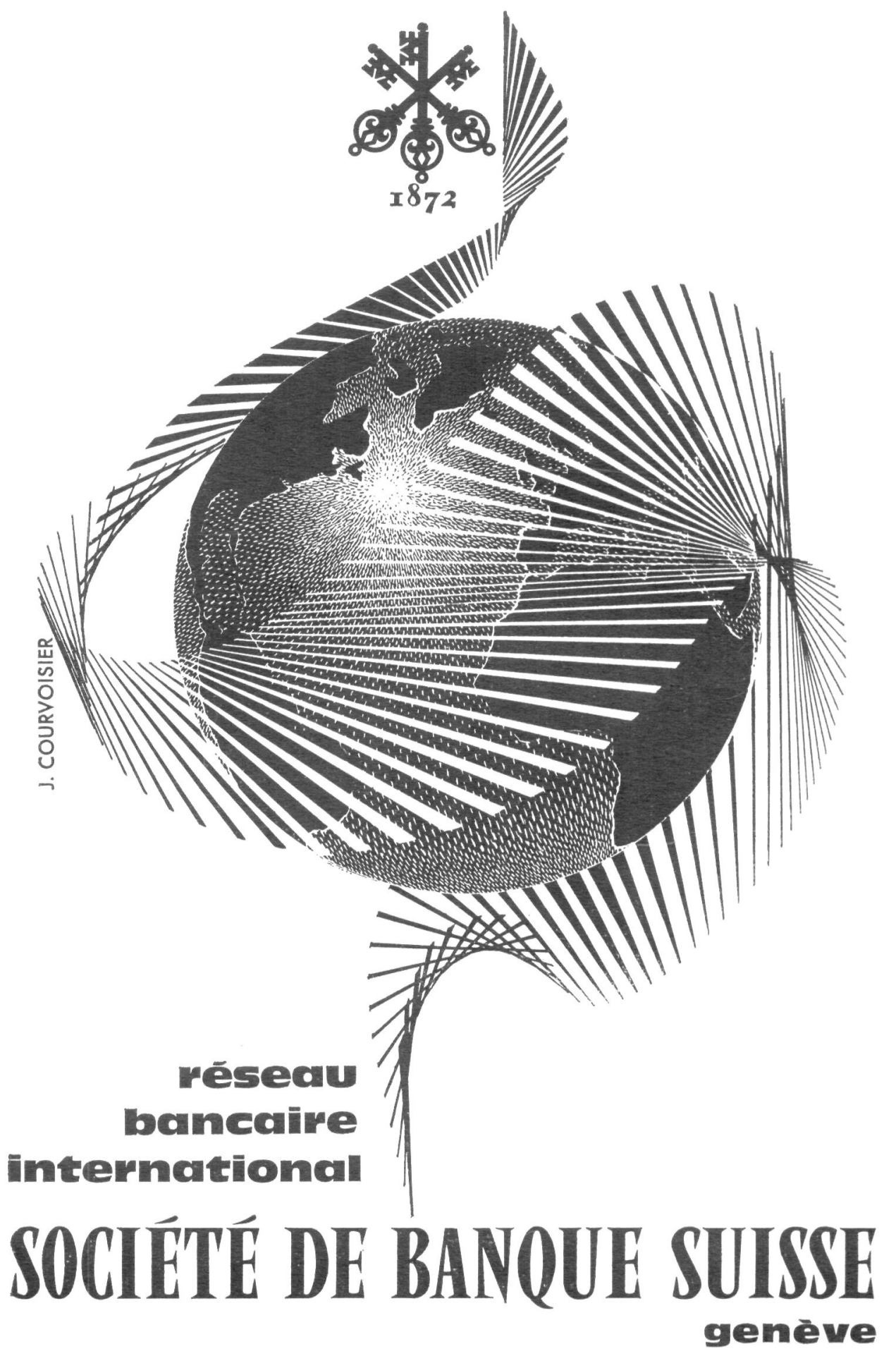




\section{SOCIETE FIDUCIAIRE ROMANDE OFOR S.A.}

9, rue d'Italie, Genève - Tél. 243293

- Expertises, revisions et organisations comptables

४ Domiciliation et administration de sociétés

$\downarrow$ Assainissements et liquidations

४ Interventions et conseils en matière fiscale

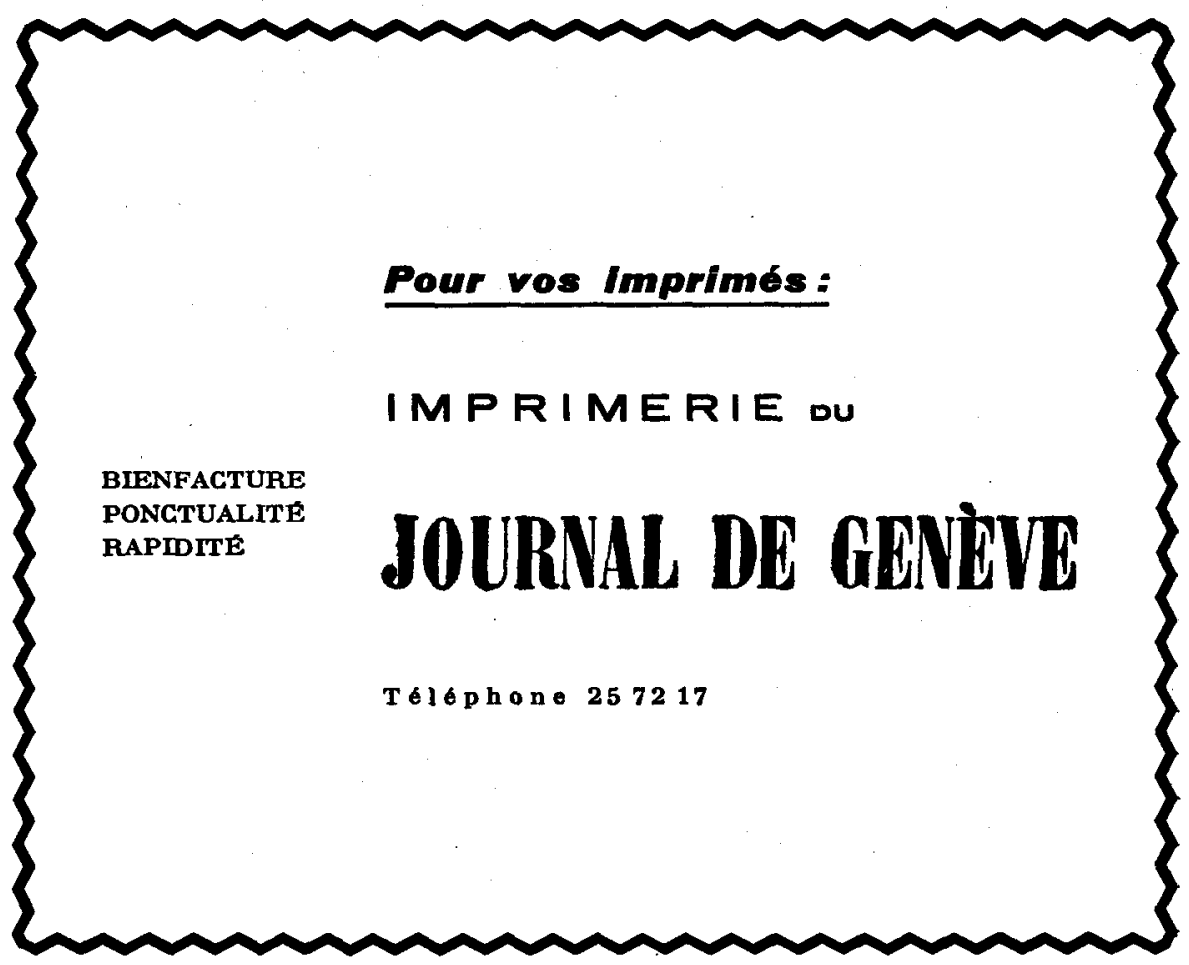




\section{REVUE INTERNATIONALE DE LA CROIX-ROUGE}

QUARANTE-DEUXIËME ANNEE - No 500

AOOT 1960

\section{SOMMAIRE}

COMITÉ INTERNATIONAL DE LA CROIX-ROUGE L'action de la Croix-Rouge au Congo . . . . . . . . . . . . 445 En faveur d'anciens prisonniers de guerre . . . . . . . . 450 Une nouvelle publication sur les Conventions de Genève . . . . . 455 Rapport d'activité du CICR . . . . . . . . . . $45^{8}$

NOUVELLES DE SOCIÉTÉS NATIONALES

Colombie . . . . . . . . . . . . . . . . 4 468 Hongrie . . . . . . . . . . . . . . . . . 440 Italie . . . . . . . . . . . . . . . . . . 474 Japon ................ . . . 480

FAITS ET DOCUMENTS

L'Année mondiale du réfugié . . . . . . . . . . . . . . . 482 L'Organisation mondiale de la Santé . . . . . . . . . . . . 486

\section{BIBLIOGRAPHIE}

\section{A TRAVERS LES REVUES}




\section{SUPPLEMENTS DE LA REVUE}

EN LANGUE ANGLAISE

Hans Hansson: The Sea rescue service in the North Sea and the Baltic. The action of the Red Cross in the Congo. - Publication of a Handbook on the Geneva Conventions.

EN LANGUE ESPAGNOLE

Hans Hansson : Los Servicios de salvamento marítimo en el mar de Norte y en el mar Báltico. - La acción de la Cruz Roja en el Congo. Una nueva publicación sobre los Convenios de Ginebra.

EN LANGUE ALIEMANDE

Hans Hansson: Seerettungsdienst in der Nordsee und Ostsee. Die Aktion des Roten Kreuzes im Kongo. - Eine neue Veröffentlichung über die Genfer Abkommen.

\section{LA}

REVUE INTERNATIONALE DE LA CROIX-ROUGE est publiée chaque mois par le Comité international de la Croix-Rouge

7, avenue de la Paix, Genève (Suisse) - Compte de chèques postaux I. 1767 Abonnement un an : Fr. 20,- ; le numéro Fr. 2,-

Pour les pays suivants : Algérie, Allemagne, Autriche, Belgique, Danemark, Finlande, France, Italie, Luxembourg, Maroc, Norvège, Pays-Bas, Portugal, Sarre, Suede, Tunisie, Vatican, on peut s'abonner aupres des bureaux de poste (fr. 8. 20,50

Rédaction : JEAN-G. LOSSIER 